\title{
Application of an Intelligent Hybrid Metaheuristic Algorithm for Multiobjective Redundancy Allocation Problem with Sustainable Maintenance
}

\author{
Tri Tjahjono, ${ }^{1}$ Dinesh Mavaluru $\mathbb{D}^{2},{ }^{2}$ Dowlath Fathima, ${ }^{3}$ Akila Thiyagarajan, ${ }^{4}$ \\ Wanich Suksatan $\mathbb{D}^{5}{ }^{5}$ Nalbiy Salikhovich Tuguz, ${ }^{6}$ Lyubov A. Melnikova, ${ }^{7}$ Ismail Husein, ${ }^{8}$ \\ Lakshmi Thangavelu, ${ }^{9}$ Yermek Abilmazhinov, ${ }^{10}$ and Mamun Habib ${ }^{11}$ \\ ${ }^{1}$ Department of Mechanical Engineering, Faculty of Engineering, Universitas Muhammadiyah Surakarta, Surakarta, \\ Jawa Tengah 57102, Indonesia \\ ${ }^{2}$ Department of Information Technology, College of Computing and Informatics, Saudi Electronic University, \\ Riyadh, Saudi Arabia \\ ${ }^{3}$ Department of Basic Sciences, College of Science and Theoretical Studies, Saudi Electronic University, Riyadh, Saudi Arabia \\ ${ }^{4}$ Department of Computer Science, College of Computer Science, King Khalid University, Alsamer, Abha, Saudi Arabia \\ ${ }^{5}$ Faculty of Nursing, HRH Princess Chulabhorn College of Medical Science, Chulabhorn Royal Academy, \\ 906 Kamphaeng Phet 6 Road, Talat Bang Khen, Lak Si, Bangkok 10210, Thailand \\ ${ }^{6}$ Department of Higher Mathematics, Kuban State Agrarian University, Krasnodar, Russia \\ ${ }^{7}$ Financial University under the Government of the Russian Federation, Moscow, Russia \\ ${ }^{8}$ Department of Mathematics, Universitas Islam Negeri Sumatera Utara, Medan, Indonesia \\ ${ }^{9}$ Department of Pharmacology, Saveetha Dental College and Hospital, Saveetha Institute of Medical and Technical Sciences, \\ Saveetha University, Chennai, India \\ ${ }^{10}$ Shakarim University of Semey, Semey, Kazakhstan \\ ${ }^{11}$ School of Business and Entrepreneurship, Independent University, Dhaka, Bangladesh
}

Correspondence should be addressed to Mamun Habib; mamunhabib@iub.edu.bd

Received 3 June 2021; Revised 19 July 2021; Accepted 7 September 2021; Published 25 September 2021

Academic Editor: Alireza Goli

Copyright (c) 2021 Tri Tjahjono et al. This is an open access article distributed under the Creative Commons Attribution License, which permits unrestricted use, distribution, and reproduction in any medium, provided the original work is properly cited.

The present study aimed to optimize the redundancy allocation problem based on sustainable maintenance. For this purpose, the goal is to design a complex system based on redundancy allocation by considering the weight and reliability criteria of the system and the maintenance and repair costs through the sustainability approach. In this regard, a mathematical model has been developed. This model minimizes system reliability and system weight simultaneously. There are also budget constraints on repair costs, environmental costs, purchase of spare parts, and energy risk costs. In order to optimize this model, a hybrid algorithm based on Whale Optimization Algorithm (WOA), Genetic Algorithm (GA), and Simulated Annealing (SA) is proposed. Accordingly, 81 test problems are provided and optimized by the proposed algorithm. The obtained numerical results indicate that, with increasing failure time of each component, the system's reliability increases and the weight of the whole system increases. Moreover, changing the Weibull distribution parameters directly affects the total amount of system reliability, but does not have a definite and accurate effect on the total weight of the system. Moreover, increasing the budget for maintenance leads to finding solutions with more reliability and less weight. 


\section{Introduction}

The redundancy allocation problem (RAP) is an essential issue in optimizing the reliability of complex high-tech, high-component systems that require a high level of reliability. Classic reliability models usually consider both healthy and faulty conditions for each of the operating components of the system. However, in recent years, in the field of reliability research, several models have been proposed considering performance measurements for each component. Accordingly, each component with different probabilities has different performance rates. In general, redundancy allocation involves adding surplus components to the system under conditions that, while increasing its reliability, optimize the designed system configuration. Since adding more components will increase the cost, volume, and weight of the system, the interaction between these factors is presented in the form of a multiobjective problem.

Sustainable maintenance in RAP was first introduced by [1]. In their paper, first, the initial system was designed; then, using the obtained information, the aspects of production program and maintenance costs and sustainability performance were evaluated. As a result, based on sustainable maintenance, the reliability of each component and the whole system are improved. The economic aspect of sustainability and customer satisfaction increase; environmental pollutions and maintenance costs were reduced by providing a reliability-based program. Adding this style of maintenance can address the challenges of maintaining reliability in redundancy allocation.

This research develops the study of [1-3]. As a novel contribution in the field of RAP, this study deals with the multiobjective optimization of redundancy allocation under maintenance with a sustainability approach. For this purpose, the system under study, in addition to complying with weight and configuration constraints, must clearly respect the views of customers' satisfaction.

As a novel contribution, it is assumed that customers have a utility function that is inversely related to the continuous failures that occur in the system. Also, the maintenance process should not lead to damage to the environment. To consider the economic perspective, the costs of designing and maintaining the system must be controlled. There are three main aspects of the proposed optimization model. (1) Minimize system design based on its weight; (2) maximize customer satisfaction based on system reliability; (3) control the pollutions, maintenance, and operational costs. Since the optimization of the problem is a challenging issue, the hybrid multiobjective solution method based on a whale optimization algorithm, genetic algorithms, and simulated annealing is used.

The main question of this research is how to present and apply a new metaheuristic algorithm to solve the redundancy allocating problem that has the best possible solution and the shortest solution time.

In the rest of the article and in Section 2, the most critical research items in this field are presented. In Section 3, the mathematical model of this research is presented, and in Section 4 , the proposed metaheuristic algorithm to solve this model is presented. In Section 5, numerical results are presented, and finally, in Section 6, conclusions are presented.

\section{Literature Review}

Lins and Droguett [4] used a multiobjective genetic algorithm and its combination with discrete simulation to solve the redundancy allocation problem. Liang and Lo [5] developed a variable neighborhood search (VNS) algorithm to solve multiobjective redundancy allocation problems. The efficiency of this algorithm was tested on three sets of problems with 5,14 , and 24 subsystems, respectively. Uysal [6] modeled four different types of multiobjective reliability optimization problems using distance values. To solve these models, an advanced genetic algorithm and the concept of the Pareto solution set have been used. Sadjadi and Soltani [7] presented a bee optimization algorithm to solve reliability optimization problems. Chambari et al. [8] developed a two-objective model of redundancy allocation. They presented the two-objective reliability in a series-parallel system with a certain level of component redundancy strategy. To solve this model, they used two NSGA-II algorithms and a multiobjective particle swarm optimization algorithm. Ghorabaee et al. [9] presented a new and efficient approach to modeling the objective function of their redundancy allocation problem. Their proposed approach is based on simulation and optimization methods through response level simulation using Enterprise Dynamic (ED) simulation software. Next, the relationship between decision and response variables has been obtained using experimental design. Ardakan and Hamadani [10] studied two-objective RAP based on maximizing the total reliability and minimizing the total volume of the system. They then used two statistical methods and comparative decision-making based on the quality of the solutions obtained and also the processing time, and finally ranked among the existing solution methods. Zhang and Chen [11] presented a mathematical model to maximizes the average operating time of the system. They used two metaheuristic algorithms and particle swarm optimization to solve their proposed redundancy allocation problem.

Salmasnia et al. [12] modeled a series-parallel redundancy allocation problem in which system reliability is maximized, and the total cost is minimized. Teimoori et al. [13] developed an electromagnetic mechanism metaheuristic algorithm to solve a redundancy allocation problem. Long et al. [14] introduced a redundancy allocation problem with multiple strategy choices in which the concepts of active redundancy and standby redundancy were considered simultaneously. They used particle swarm motion optimization to optimize their problem. Karevan and Vasili [1] presented a two-objective model for the redundancy allocation problem. In this model, reliability, as well as various maintenance and environmental costs, are optimized. Budget constraints are also included in the allocation of redundancy. Ouyang et al. [3] proposed a reliability-RAP problem with considering standby components and cold strategy. Moreover, they applied an improved version of the 
particle swarm optimization algorithm with a flexible design. Zaretalab et al. [15] considered a multistate component in RAP. In this research, the reliability of the supplier is investigated. In order to find the optimal solution, they proposed a mathematical model with the objective to maximize the total reliability and used a Memetic algorithm to solve it. Reference [16] proposed a multiobjective mathematical model for a multitype system. In this model, the optimal redundancy strategy is obtained by using the NSGA-II algorithm. RAP.

Table 1 reviews the most important articles in the field of

After reviewing various research on redundancy RAP optimization, it is clear that the most important research gap in this area is the optimization of redundancy allocation under preventive maintenance with a sustainability approach. Therefore, as a novel contribution, the present study optimizes the redundancy allocation problem based on sustainable maintenance. For this purpose, the goal is to design a complex system under conditions that, in addition to considering the weight and reliability criteria of the system, the maintenance and repair costs of the system are controlled through the sustainability approach. Moreover, a novel metaheuristic approach based on WOA, GA, and SA is proposed which is a specific contribution in the field of RAP optimization.

\section{Research Mathematical Model}

In this research, a series of parallel series elements that contain $k$ components are considered in a way that can be used for each component of a number of spare parts as parallel. For this purpose, it is assumed that for the first part $n_{1}$ spare part, for the second part $n_{2}$ spare part up to $k_{m}$ part, $n_{k}$ spare parts can be used. Figure 1 shows such a structure.

Accordingly, there is a specific operational budget because each of the spare parts has different prices. Also, the weight of the system should not be increased from a certain limit.

It is assumed that the probabilistic function for each component in the series-parallel structure follows the Weibull distribution. According to equation (1), the reliability of the part $i(i \in\{1,2, \ldots, k\})$ in time $t$ is calculated as follows:

$$
R_{i}=e^{\left(t / \theta_{i}\right) \beta_{i}}, \quad i \in\{1,2, \ldots, k\}
$$

where the parameters $\beta_{i}$ and $\theta_{i}$ are the shape and scale parameters of the $i_{m}$ component, respectively. Next, according to equation (2), the reliability of a set of components of type $i_{m}$ when $n_{i}$ spare items are added is calculated as follows:

$$
R_{i}^{T}=1-\left(1-R_{i}\right)^{n_{i}+1}, \quad i \in\{1,2, \ldots, k\} .
$$

Next, based on equation (3), the total reliability of the series-parallel system can be calculated:

$$
R_{i}^{T}=\prod_{i=1}^{k}\left(1-\left(1-R_{i}\right)^{n_{i}+1}\right), \quad i \in\{1,2, \ldots, k\} .
$$

In equations (2) and (3), it was assumed that spare parts would be applied to all components. To solve this challenge, suppose the integer variable $x_{i}$ represents the number of spare parts that can be used for component $i_{m}$. In this case, equations (4) and (5) are rewritten as follows. In this case, equations (4) and (5) are rewritten as follows:

$$
\begin{aligned}
& R_{i}^{T}=1-\left(1-R_{i}\right)^{x_{i}+1}, \quad i \in\{1,2, \ldots, k\}, \\
& R_{i}^{T}=\prod_{i=1}^{k}\left(1-\left(1-R_{i}\right)^{x_{i}+1}\right), \quad i \in\{1,2, \ldots, k\} .
\end{aligned}
$$

After stating the structure of the reliability calculation, the mathematical model of the redundancy allocation problem without considering permanent maintenance will be as follows:

$$
\max R_{i}^{T}=\prod_{i=1}^{k}\left(1-\left(1-R_{i}\right)^{x_{i}+1}\right) .
$$

Subject to

$$
\begin{aligned}
R_{i} & =e^{-\left(t^{\beta_{i}} / \theta_{i}\right)}, \quad i \in\{1,2, \ldots, k\}, \\
\sum_{i=1}^{k} c_{i} x_{i} & \leq \beta^{c},
\end{aligned}
$$

$$
\sum_{i=1}^{k} w_{i}\left(x_{i}+1\right) \leq \beta^{w}, \quad x_{i} \in\left\{0,1, \ldots, n_{i}\right\} .
$$

In the presented basic mathematical model, the values of $c_{i}$ and $w_{i}$ are the purchase cost and the weight, respectively, except for type $i$. The values $\beta^{c}$ and $\beta^{w}$ also show the budgets associated with the maximum justified cost for the system and the maximum acceptable weight for the whole system. Equation (6) is the model's objective, which aims to maximize the reliability of the whole system. Equation (7) calculates the reliability of each component. Equation (8) is required to consider the weight of the original components in the calculation of the overall weight of the system. But this is not needed to limit the budget deficit in equation (9) since the budget is considered only for the purchase of spare parts and should not be considered the existence of the original components. In order to develop the presented mathematical model and reflect preventive maintenance, it is assumed that $\alpha_{i}$ percentage of components type $i$ may need to be replaced. Accordingly, the fixed cost of reinstallation is calculated based on the following:

$$
R A_{E C O 1(t)}=\frac{\sum_{i=1}^{k} C_{i}\left(1-R_{i}\right)^{y_{i}}}{\left(1+r_{m}\right)^{t}},
$$

where $y_{i}=\alpha_{i} n_{i}$ and $r_{m}$ is the discount rate. $C_{i}$ is the fixed cost of supplying a spare part for the Type $i$ part. Equation (11) represents the second economic aspect. This equation identifies the variable costs of supply spare parts:

$$
R A_{E C O 2(t)}=\frac{\sum_{i=1}^{k}\left(1-R_{i}\right)^{y_{i}} *\left[\left(n_{i} * \mathrm{Cust}_{i}\right)\right]}{\left(1+r_{m}\right)^{t}},
$$


TABLE 1: The summary of the reviewed literature.

\begin{tabular}{|c|c|c|c|c|c|c|c|c|}
\hline Research & $\begin{array}{c}\text { Budget } \\
\text { constraint }\end{array}$ & $\begin{array}{c}\text { Weight } \\
\text { constraint }\end{array}$ & $\begin{array}{c}\text { Single } \\
\text { objective }\end{array}$ & Multiobjective & $\begin{array}{c}\text { Corrective } \\
\text { maintenance }\end{array}$ & $\begin{array}{c}\text { Preventive } \\
\text { maintenance }\end{array}$ & Sustainability & Solution method \\
\hline $\begin{array}{l}\text { Sahoo et al. } \\
{[17]}\end{array}$ & & $\sqrt{ }$ & & $\sqrt{ }$ & & & & $\begin{array}{l}\text { Improved genetic } \\
\text { algorithm }\end{array}$ \\
\hline $\begin{array}{l}\text { Sadjadi and } \\
\text { Soltani [7] }\end{array}$ & $\sqrt{ }$ & $\sqrt{ }$ & & $\sqrt{ }$ & $\sqrt{ }$ & & & $\begin{array}{c}\text { Bee colony } \\
\text { optimization }\end{array}$ \\
\hline $\begin{array}{l}\text { Abouei } \\
\text { Ardakan } \\
\text { et al. [10] }\end{array}$ & $\sqrt{ }$ & & $\sqrt{ }$ & & & & $\sqrt{ }$ & $\begin{array}{l}\text { Differential } \\
\text { evolution }\end{array}$ \\
\hline $\begin{array}{l}\text { Zhang and } \\
\text { Chen [11] }\end{array}$ & & $\sqrt{ }$ & $\sqrt{ }$ & & & & $\sqrt{ }$ & $\begin{array}{l}\text { Particle swarm } \\
\text { optimization }\end{array}$ \\
\hline $\begin{array}{l}\text { Chambari } \\
\text { et al. [8] }\end{array}$ & & $\sqrt{ }$ & & $\sqrt{ }$ & $\sqrt{ }$ & & & NSGA-II \\
\hline $\begin{array}{l}\text { Lins and } \\
\text { Droguett [4] }\end{array}$ & $\sqrt{ }$ & & & $\sqrt{ }$ & & $\sqrt{ }$ & & Genetic algorithm \\
\hline $\begin{array}{l}\text { Liang and Lo } \\
\text { [5] }\end{array}$ & $\sqrt{ }$ & & & $\sqrt{ }$ & & & & $\begin{array}{c}\text { Variable } \\
\text { neighborhood } \\
\text { search }\end{array}$ \\
\hline $\begin{array}{l}\text { Long et al. } \\
{[14]}\end{array}$ & & & & $\sqrt{ }$ & $\sqrt{ }$ & & & $\begin{array}{l}\text { Particle swarm } \\
\text { optimization }\end{array}$ \\
\hline $\begin{array}{l}\text { Karevan and } \\
\text { Vasili [1] }\end{array}$ & $\sqrt{ }$ & & & $\sqrt{ }$ & & $\sqrt{ }$ & $\sqrt{ }$ & $\begin{array}{l}\text { Multiobjective } \\
\text { particle swarm } \\
\text { optimization }\end{array}$ \\
\hline $\begin{array}{l}\text { Ouyang et al. } \\
\text { [3] }\end{array}$ & & & $\sqrt{ }$ & & $\sqrt{ }$ & & & $\begin{array}{l}\text { Particle swarm } \\
\text { optimization }\end{array}$ \\
\hline $\begin{array}{l}\text { Zaretalab } \\
\text { et al. [15] }\end{array}$ & & $\sqrt{ }$ & $\sqrt{ }$ & & & $\sqrt{ }$ & & Memetic algorithm \\
\hline $\begin{array}{l}\text { Wang et al. } \\
{[16]}\end{array}$ & $\sqrt{ }$ & $\sqrt{ }$ & & $\sqrt{ }$ & & & & NSGA-II \\
\hline $\begin{array}{l}\text { Present } \\
\text { research }\end{array}$ & $\sqrt{ }$ & $\sqrt{ }$ & & $\sqrt{ }$ & & $\sqrt{ }$ & $\sqrt{ }$ & $\begin{array}{l}\text { Hybrid whale- } \\
\text { genetic simulated } \\
\text { annealing }\end{array}$ \\
\hline
\end{tabular}

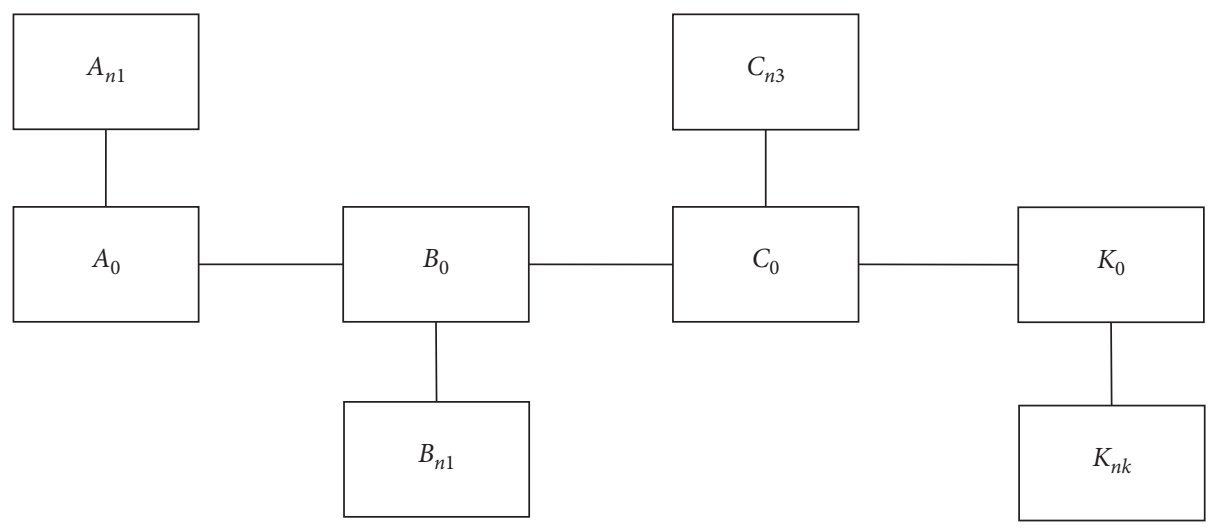

FIGURE 1: The proposed RAP series-parallel system structure.

where Cust ${ }_{i}$ is related to the variable cost of each component of type $i$. In equation (12), mean downtime (MDT) is used and indicates the time to find fault and fix it. As a result, equation (12) shows the total lost amount of time:

$$
R A_{S O C 1(t)}=\sum_{i=1}^{k}\left(1-R_{i}\right)^{y_{i}} * M D T * y_{i} .
$$

Equation (13) shows the sum of the total cost of the customer dissatisfaction resulting from the suspension of the system. In this equation, $c h_{i}$ is the cost of customer dissatisfaction is due to each hour of system downtime:

$$
R A_{S O C 2(t)}=\operatorname{ch} * R A_{S O C 1(t)}=\sum_{i=1}^{k}\left(1-R_{i}\right)^{y_{i}} * \operatorname{ch} * M D T * y_{i} .
$$

Equations (14) and (15) represent carbon emission cost and the amount of energy cost, respectively. In these equations, $\mathrm{CD}$ is the carbon emission cost for each repair 
activity and EC represents the cost of consumed energy for each repair activity.

$$
\begin{aligned}
& R A_{E N V 1(t)}=\sum_{i=1}^{k}\left(1-R_{i}\right)^{y_{i}} * C D, \\
& R A_{E N V 2(t)}=\sum_{i=1}^{k}\left(1-R_{i}\right)^{y_{i}} * E C .
\end{aligned}
$$

Based on the above equations, the developed multiobjective mathematical model of redundancy allocation with respect to preventive maintenance can be presented as follows:

$$
\begin{aligned}
& \max f_{1}=\prod_{i=1}^{k}\left(1-\left(1-R_{i}\right)^{x_{i}+1}\right), \\
& \min f_{2}=\sum_{i=1}^{k} w_{i}\left(x_{i}+1\right) .
\end{aligned}
$$

Subject to

$$
\begin{aligned}
R_{i}= & e^{-\left(t^{\beta_{i}} / \theta_{i}\right)}, \quad i \in\{1,2, \ldots, k\}, \\
& \sum_{i=1}^{k} c_{i} x_{i}+R A_{E C O 1(t)}+R A_{E C O 2(t)}+R A_{S O C 2(t)} \\
& +R A_{E N V 1(t)}+R A_{E N V 2(t) \leq \beta^{c}} \\
R A_{E C O 1(t)}= & \frac{\sum_{i=1}^{k} C_{i}\left(1-R_{i}\right)^{y_{i}}}{\left(1+r_{m}\right)^{t}}, \\
R A_{E C O 2(t)}= & \frac{\sum_{i=1}^{k}\left(1-R_{i}\right)^{y_{i}} *\left[\left(n_{i} * \mathrm{Cust} t_{i}\right)\right]}{\left(1+r_{m}\right)^{t}} \\
R A_{S O C 2(t)}= & \frac{\sum_{i=1}^{k}\left(1-R_{i}\right)^{y_{i}} * c h * M D T * y_{i}}{\left(1+r_{m}\right)^{t}}, \\
R A_{E N V 1(t)}= & \frac{\sum_{i=1}^{k}\left(1-R_{i}\right)^{y_{i}} * C D}{\left(1+r_{m}\right)^{t}}, \\
R A_{E N V 2(t)}= & \sum_{i=1}^{k}\left(1-R_{i}\right)^{y_{i}} * E C, \quad x_{i} \in\left\{0,1, \ldots, n_{i}\right\} .
\end{aligned}
$$

\section{Hybrid Whale-Genetic-Simulated Annealing Algorithm}

In order to present the proposed hybrid algorithm, first, the whale optimization algorithm (WOA) is described, which is one of the newest presented metaheuristic algorithms. The WOA algorithm is one of the nature-inspired and population-based optimization algorithms that can be used in various fields [18]. The details of this metaheuristic algorithm are explained as follows.
The whales can identify the location of the chase and surround them. In WOA, each solution is interpreted as a whale with a specific position. Since the optimal design space is not known in the search space, the algorithm assumes that the best candidate solution for the present is the target hunt or is close to the desired state. After the best search factor is identified, other search factors try to update their location relative to the best search factor. This behavior is expressed through the following equations:

$$
\begin{aligned}
\vec{D} & =\left|C \cdot X^{*}(t)-X(t)\right|, \\
X(t+1) & =X^{*}(t)-\vec{A} \cdot \vec{D},
\end{aligned}
$$

where $t$ denotes the current iteration, $A$ and $C$ are the coefficient vectors, $X^{*}$ the location vector is the best solution obtained now, and $X$ the location vector of the current solution. It should be noted that if there is a better solution, $X^{*}$ should be updated in each iteration. Vectors $A$ and $C$ are calculated as follows:

$$
\begin{aligned}
& \vec{A}=2 \vec{a} \cdot \vec{r}-\vec{a}, \\
& \vec{C}=2 \cdot \vec{r},
\end{aligned}
$$

where $a$ is a controllable parameter that linearly decreases from 2 to 0 and $r$ is a random vector at a distance of 0 to 1 .

In this research, a solution method based on whale optimization, genetics, and simulated annealing algorithms is developed and called HWGSA to extract the nondominated solutions of the proposed mathematical model. In this hybrid algorithm, siege hunting is used in the WOA algorithm to generate neighbor solutions. Moreover, the crossover operator in the GA algorithm is used to converge the solutions (exploration). Finally, to create variation in the solutions, the neighborhood creation operator is used in the SA algorithm (exploitation).

\subsection{The HWGSA Steps}

Step 1 (solution structure): in the HWGSA algorithm, the number of spare parts with $K$ different component is displayed in the form of a vector as shown in Figure 2. In this vector, each cell demonstrates the number of allocation redundant component to the system.

According to Figure 2, $n_{1}$ is the number of redundant spare parts allocated to the first item of the system. Other cells can be defined in the same way. By using this solution representation, it can be a guarantee that all decision variables and the model constraints can be handled in the proposed algorithm.

Step 2: randomly generate POP initial solutions and calculate the values of the objective functions $\left(f_{1}, f_{2}\right)$ for each member of the population based on equations (16) and (17).

Step 3: generate a set of new solutions based on equations (19)-(22) in the WOA algorithm and set them into $P$ set. 


\begin{tabular}{|l|l|l|l|}
\hline$n_{1}$ & $n_{2}$ & $\cdots$ & $n_{k}$ \\
\hline
\end{tabular}

Figure 2: Solution representation.

Step 4: repeat steps 5 to 19 for $I T_{\max }$ times

Step 5: for each solution $(S)$ in the POP, set $k \longleftarrow 0$ and $y \longleftarrow 0$ and go to step 6 .

Step 6: compare $S$ with each solution $(j)$ in Pset.

Step 6.1: if $f_{1}(S) \geq f_{1}^{P \text { set }}(j)$ and $f_{2}(S) \leq f_{2}^{P \text { set }}(j)$,

$$
\text { Pset } \longleftarrow \frac{P \text { set }}{j} \text {. }
$$

Step 6.2: else, if $f_{1}(S)>f_{1}^{P s e t}(j)$ or $f_{2}(S)<f_{2}^{P s e t}(j)$,

$$
k \longleftarrow k+1 \text {. }
$$

Step 6.3: else, if $f_{1}(S) \leq f_{1}^{P \text { set }}(j)$ or $f_{2}(S) \geq f_{2}^{P \text { set }}(j)$,

$$
y \longleftarrow y+1 \text {. }
$$

Step 6.4: if $k>0$ and $y==0$,

$$
\text { Pset } \longleftarrow \text { Pset } \cup S \text {. }
$$

Step 7: for each solution: $S_{i}=\left\{n_{1 i}, n_{2 i}, \ldots, n_{k i}\right\}$ and $S_{j}=$ $\left\{n_{1 j}, n_{2 j}, \ldots, n_{k j}\right\}$ from $P$ set, repeat step 8 .

Step 8: calculate the new $S^{\text {new }}$ solution using the crossover operator in the GA algorithm:

$S^{\text {new }} \longleftarrow\left\{<\left(n_{1 i} * n_{1 j}\right)^{0.5>} \cup<\left(n_{2 i} * n_{2 j}\right)^{0.5>} \cup, \ldots, \cup<\left(n_{k i} * n_{k j}\right)^{0.5>}\right\}$

Step 9: $T \longleftarrow T^{\max }$.

Step 10: repeat steps 11-16 while $T>T^{\mathrm{min}}$ (based on SA algorithm).

Step 11: generate a random number in the range $\{1,2, \ldots, 2 K\}$.

Step 12: if $a==2 * i \in\{1,2, \ldots, 2 K\}$.

Generate a random $b$ number $b$ in the range $b \in\left[1, n_{i}^{\max }\right]$.

Step 12.1: if $n_{i}+b \leq n_{i}^{\max }$, then

$$
S^{\text {new }}\left\{n_{i}\right\} \longleftarrow S^{\text {new }}\left\{n_{i}\right\}+b .
$$

Step 13: if $a==2 * i-1$, thengenerate a random $b$ number $b$ in the range $b \in\left[1, n_{i}^{\max }\right]$.

Step: 13.1: if $S^{\text {new }}\left\{n_{i}\right\}-b \geq 0$,

$$
S^{\text {new }}\left\{n_{i}\right\} \longleftarrow S^{\text {new }}\left\{n_{i}\right\}-b .
$$

Step 14: for $S^{\text {new }}$, calculate the values of the objective functions $f_{1}, f_{2}$ based on equations (16) and (17).

Step 15: if $f_{1}\left(S^{\text {new }}\right) \geq f_{1}(S)$ and $f_{2}\left(S^{\text {new }}\right) \leq f_{2}(S)$,

$$
S \longleftarrow S^{\text {new }} \text {. }
$$

Step 15.1: if $f_{1}\left(S^{\text {new }}\right)>f_{1}(S)$ or $f_{2}\left(S^{\text {new }}\right)<f_{2}(S)$,

$$
\begin{aligned}
& \Delta e_{1} \longleftarrow f_{1}(S)-f_{1}\left(S^{\text {new }}\right), \\
& \Delta E \longleftarrow f_{2}\left(S^{\text {new }}\right)-f_{2}(S), \\
& \Delta E \longleftarrow \lambda_{1} \Delta e_{1}+\lambda_{2} \Delta e_{2}, \\
& \lambda_{1}=1 \text { whether }\left(\Delta e_{1}>0\right) \text { or } 0\left(\Delta e_{1} \leq 0\right), \\
& \lambda_{2}=1 \text { whether }\left(\Delta e_{2}>0\right) \text { or } 0\left(\Delta e_{2} \leq 0\right), \\
& A \longleftarrow e^{-(\Delta E / K T)} .
\end{aligned}
$$

Step 15.2: if random $<A$,

$$
S \longleftarrow S^{\text {new }}
$$

Step 16: $T \longleftarrow T * \delta$ and return to step 10 .

Step 17: NewPOP $\longrightarrow$ NewPOP $\cup S^{\text {new }}$

Step 18: insert POP $\longrightarrow$ NewPOP and go to step 4 .

Step 19: display the $P_{\text {set }}$ set.

In order to adjust the parameters of the algorithm, several problems have been randomly generated and optimized. Then, with the trial and error approach, the best values for the algorithm parameters are selected. $I T_{\max }=100, A=0.3$, and $T^{\min }=1$ are set accordingly.

\section{Numerical Results}

First, it is necessary to examine the results of the algorithm by solving an important case study in Iran. In this case study, a system with 5 components is available. The reliability of each component in this system is measured based on the Weibull distribution with the beta and theta parameters, which have values of 2 and 10, respectively. According to the previous data, up to $30 \%$ of the parts are damaged in each component. The average failure time is estimated at 2 days. The cost of providing each spare part is 5 currency units and the repair fee is equal to 3.5 currency units. The cost of customer dissatisfaction with each day of part failure is equivalent to 1 currency. The interest rate is $10 \%$ and the maximum maintenance budget is 200 currency units. Regarding environmental pollution, the amount of $\mathrm{CO} 2$ produced per day of system activity is equal to 1.7 units and the amount of energy consumed per day is equal to 1.5 units.

With this information and the design of the HWGSA algorithm in the MATLAB R2016, the redundancy allocation problem is optimized. After executing the HWGSA algorithm, a set of Pareto solutions is obtained, which is shown in Figure 3.

As shown in Figure 3, the value of the first objective function varies between 0.05 and 0.45 . The value of the second objective function fluctuates between 120 and 320 . Since the first objective function is of the maximization type, the ideal value for it is 0.45 . Also, since the second objective function is of the minimization type, the ideal value for it is 120. When the first objective function moves from 0.05 to its ideal value, the second objective function moves away from its ideal value. In other words, in the set of Pareto solutions obtained, it is never possible to approach both goals at the 


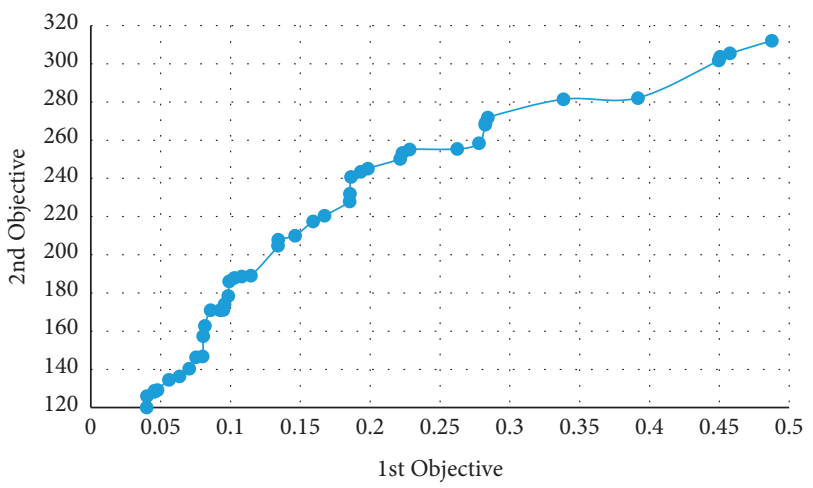

Figure 3: The obtained Pareto solutions from the proposed hybrid algorithm.

TABLE 2: The information of the generated test problems.

\begin{tabular}{|c|c|c|c|c|}
\hline Test problem & MDT & $\theta_{i}$ & $\beta_{i}$ & $\beta^{c}$ \\
\hline$p 1$ & 2 & 3 & 2 & 150 \\
\hline$p 2$ & 4 & 3 & 2 & 150 \\
\hline p3 & 6 & 3 & 2 & 150 \\
\hline$p 4$ & 2 & 7 & 2 & 150 \\
\hline$p 5$ & 4 & 7 & 2 & 150 \\
\hline$p 6$ & 6 & 7 & 2 & 150 \\
\hline p7 & 2 & 12 & 2 & 150 \\
\hline$p 8$ & 4 & 12 & 2 & 150 \\
\hline$p 9$ & 6 & 12 & 2 & 150 \\
\hline$p 10$ & 2 & 3 & 3 & 150 \\
\hline$p 11$ & 4 & 3 & 3 & 150 \\
\hline$p 12$ & 6 & 3 & 3 & 150 \\
\hline$p 13$ & 2 & 7 & 3 & 150 \\
\hline p14 & 4 & 7 & 3 & 150 \\
\hline$p 15$ & 6 & 7 & 3 & 150 \\
\hline$p 16$ & 2 & 12 & 3 & 150 \\
\hline$p 17$ & 4 & 12 & 3 & 150 \\
\hline$p 18$ & 6 & 12 & 3 & 150 \\
\hline p19 & 2 & 3 & 4 & 150 \\
\hline$p 20$ & 4 & 3 & 4 & 150 \\
\hline$p 21$ & 6 & 3 & 4 & 150 \\
\hline$p 22$ & 2 & 7 & 4 & 150 \\
\hline p23 & 4 & 7 & 4 & 150 \\
\hline p24 & 6 & 7 & 4 & 150 \\
\hline p25 & 2 & 12 & 4 & 150 \\
\hline p26 & 4 & 12 & 4 & 150 \\
\hline$p 27$ & 6 & 12 & 4 & 150 \\
\hline$p 28$ & 2 & 3 & 2 & 200 \\
\hline p29 & 4 & 3 & 2 & 200 \\
\hline p30 & 6 & 3 & 2 & 200 \\
\hline p31 & 2 & 7 & 2 & 200 \\
\hline p32 & 4 & 7 & 2 & 200 \\
\hline p33 & 6 & 7 & 2 & 200 \\
\hline p34 & 2 & 12 & 2 & 200 \\
\hline p35 & 4 & 12 & 2 & 200 \\
\hline p36 & 6 & 12 & 2 & 200 \\
\hline p37 & 2 & 3 & 3 & 200 \\
\hline p38 & 4 & 3 & 3 & 200 \\
\hline p39 & 6 & 3 & 3 & 200 \\
\hline$p 40$ & 2 & 7 & 3 & 200 \\
\hline$p 41$ & 4 & 7 & 3 & 200 \\
\hline
\end{tabular}


TABLE 2: Continued.

\begin{tabular}{|c|c|c|c|c|}
\hline Test problem & MDT & $\theta_{i}$ & $\beta_{i}$ & $\beta^{c}$ \\
\hline$p 42$ & 6 & 7 & 3 & 200 \\
\hline$p 43$ & 2 & 12 & 3 & 200 \\
\hline$p 44$ & 4 & 12 & 3 & 200 \\
\hline$p 45$ & 6 & 12 & 3 & 200 \\
\hline$p 46$ & 2 & 3 & 4 & 200 \\
\hline$p 47$ & 4 & 3 & 4 & 200 \\
\hline$p 48$ & 6 & 3 & 4 & 200 \\
\hline$p 49$ & 2 & 7 & 4 & 200 \\
\hline$p 50$ & 4 & 7 & 4 & 200 \\
\hline$p 51$ & 6 & 7 & 4 & 200 \\
\hline$p 52$ & 2 & 12 & 4 & 200 \\
\hline p53 & 4 & 12 & 4 & 200 \\
\hline p54 & 6 & 12 & 4 & 200 \\
\hline p55 & 2 & 3 & 2 & 350 \\
\hline p56 & 4 & 3 & 2 & 350 \\
\hline$p 57$ & 6 & 3 & 2 & 350 \\
\hline p58 & 2 & 7 & 2 & 350 \\
\hline p59 & 4 & 7 & 2 & 350 \\
\hline$p 60$ & 6 & 7 & 2 & 350 \\
\hline$p 61$ & 2 & 12 & 2 & 350 \\
\hline$p 62$ & 4 & 12 & 2 & 350 \\
\hline p63 & 6 & 12 & 2 & 350 \\
\hline$p 64$ & 2 & 3 & 3 & 350 \\
\hline$p 65$ & 4 & 3 & 3 & 350 \\
\hline p66 & 6 & 3 & 3 & 350 \\
\hline$p 67$ & 2 & 7 & 3 & 350 \\
\hline p68 & 4 & 7 & 3 & 350 \\
\hline p69 & 6 & 7 & 3 & 350 \\
\hline$p 70$ & 2 & 12 & 3 & 350 \\
\hline$p 71$ & 4 & 12 & 3 & 350 \\
\hline$p 72$ & 6 & 12 & 3 & 350 \\
\hline$p 73$ & 2 & 3 & 4 & 350 \\
\hline$p 74$ & 4 & 3 & 4 & 350 \\
\hline p75 & 6 & 3 & 4 & 350 \\
\hline p76 & 2 & 7 & 4 & 350 \\
\hline$p 77$ & 4 & 7 & 4 & 350 \\
\hline p78 & 6 & 7 & 4 & 350 \\
\hline p79 & 2 & 12 & 4 & 350 \\
\hline$p 80$ & 4 & 12 & 4 & 350 \\
\hline$p 81$ & 6 & 12 & 4 & 350 \\
\hline
\end{tabular}

same time to their ideal values. This indicates that the goals used are in conflict with each other. Proof of such a conflict confirms that the multiobjective consideration of the mathematical model is quite correct and logical and that the use of the HWGSA algorithm can well find Pareto's set of solutions to this problem.

5.1. Results of Implementation. In this part of the research, various test problems of the proposed mathematical model are optimized in order to determine the effect of different parameters of the problem on the optimal solutions of the model. In this regard, the information provided in Section 5 is used. Also, for four important parameters of the mathematical model, namely $\beta_{c}, \beta_{i}, \theta_{i}$, and MDT, 3 values each are proposed. Therefore, a total of 81 test problems have been designed. Details of each of these issues are provided in Table 2 .
After designing 81 different numerical examples, each of them was optimized in Matlab software using the HWGSA algorithm. After execution, a set of Pareto solutions to each test problem was obtained. Then, the mean values of the first and second objective functions of the Pareto solutions and also the number of Pareto solutions were reported. This report is in accordance with Table 3.

5.2. Numerical Analysis. The first parameter studied is the average failure time for which the values of 2, 4, and 6 days were considered. Figures 4 and 5 show the effect of the value of this parameter on the mean of the first objective function and the second objective function.

As shown in Figure 4, the longer the failure time, the higher the average system reliability. The reason for this is that the longer the failure time, the more parts are allocated to each component. Accordingly, to the system reliability 
TABLE 3: The results of implementing test problems.

\begin{tabular}{|c|c|c|c|}
\hline Test problem & Number of Pareto solutions & Average of the first objective & Average of the second objective \\
\hline$p 1$ & 47 & $4.63 E-08$ & 7778 \\
\hline$p 2$ & 50 & $1.90 E-07$ & 10418 \\
\hline p3 & 43 & $4.44 E-07$ & 11685 \\
\hline$p 4$ & 47 & $4.00 E-08$ & 7806 \\
\hline p5 & 50 & $1.53 E-07$ & 10012 \\
\hline$p 6$ & 50 & $4.35 E-07$ & 11987 \\
\hline p7 & 50 & $3.68 E-08$ & 7400 \\
\hline$p 8$ & 50 & $1.17 E-07$ & 9065 \\
\hline$p 9$ & 47 & $3.98 E-07$ & 11974 \\
\hline$p 10$ & 49 & 0.01662008 & 8835 \\
\hline$p 11$ & 48 & 0.03045951 & 9929 \\
\hline p12 & 49 & 0.072815946 & 13343 \\
\hline$p 13$ & 49 & 0.013728675 & 8404 \\
\hline p14 & 48 & 0.037327078 & 11058 \\
\hline p15 & 48 & 0.063445359 & 12667 \\
\hline p16 & 48 & 0.010736866 & 7748 \\
\hline p17 & 47 & 0.026810576 & 9781 \\
\hline$p 18$ & 50 & 0.053703995 & 12076 \\
\hline p19 & 50 & 0.226867023 & 8415 \\
\hline$p 20$ & 50 & 0.355636589 & 10608 \\
\hline$p 21$ & 48 & 0.476344384 & 13027 \\
\hline$p 22$ & 49 & 0.199889028 & 7810 \\
\hline$p 23$ & 49 & 0.323638156 & 9890 \\
\hline$p 24$ & 49 & 0.449719356 & 12334 \\
\hline$p 25$ & 50 & 0.212840018 & 8075 \\
\hline p26 & 50 & 0.315307874 & 9771 \\
\hline p27 & 45 & 0.42569378 & 11862 \\
\hline$p 28$ & 45 & $4.10 E-13$ & 7575 \\
\hline p29 & 47 & $1.71 E-12$ & 10087 \\
\hline p30 & 50 & $4.54 E-12$ & 12018 \\
\hline p31 & 50 & $3.73 E-13$ & 7751 \\
\hline p32 & 48 & $1.35 E-12$ & 9494 \\
\hline$p 33$ & 45 & $4.38 E-12$ & 12209 \\
\hline p34 & 49 & $3.63 E-13$ & 7710 \\
\hline p35 & 45 & $1.20 E-12$ & 9478 \\
\hline p36 & 49 & $3.00 E-12$ & 10554 \\
\hline p37 & 50 & 0.00017731 & 8309 \\
\hline p38 & 47 & 0.000592272 & 10591 \\
\hline p39 & 50 & 0.001369745 & 12721 \\
\hline$p 40$ & 50 & 0.000179898 & 8621 \\
\hline$p 41$ & 46 & 0.000445769 & 9745 \\
\hline$p 42$ & 46 & 0.001063624 & 11771 \\
\hline$p 43$ & 46 & 0.000103981 & 7192 \\
\hline$p 44$ & 50 & 0.000419827 & 9963 \\
\hline$p 45$ & 49 & 0.001049651 & 11854 \\
\hline$p 46$ & 48 & 0.03452181 & 8191 \\
\hline$p 47$ & 47 & 0.081674209 & 10598 \\
\hline$p 48$ & 47 & 0.152745337 & 13447 \\
\hline$p 49$ & 47 & 0.031635127 & 7964 \\
\hline$p 50$ & 50 & 0.060361023 & 9335 \\
\hline p51 & 50 & 0.133430003 & 12419 \\
\hline p52 & 47 & 0.031239782 & 7892 \\
\hline p53 & 47 & 0.065909873 & 9920 \\
\hline$p 54$ & 45 & 0.130330611 & 12522 \\
\hline$p 55$ & 50 & $3.64 E-18$ & 7555 \\
\hline$p 56$ & 49 & $1.37 E-17$ & 9683 \\
\hline$p 57$ & 46 & $4.26 E-17$ & 12421 \\
\hline p58 & 49 & $3.18 E-18$ & 7753 \\
\hline p59 & 48 & $1.18 E-17$ & 9760 \\
\hline$p 60$ & 46 & $3.71 E-17$ & 12045 \\
\hline
\end{tabular}


TABLE 3: Continued.

\begin{tabular}{lccc}
\hline Test problem & Number of Pareto solutions & Average of the first objective & Average of the second objective \\
\hline$p 61$ & 47 & $3.12 E-18$ & 7544 \\
$p 62$ & 50 & $8.92 E-18$ & 9106 \\
$p 63$ & 47 & $2.76 E-17$ & 11049 \\
$p 64$ & 47 & $1.13 E-06$ & 7768 \\
$p 65$ & 50 & $3.99 E-06$ & 9129 \\
$p 66$ & 50 & $1.37 E-05$ & 12406 \\
$p 67$ & 50 & $1.32 E-06$ & 8025 \\
$p 68$ & 45 & $3.78 E-06$ & 9788 \\
$p 69$ & 50 & $1.11 E-05$ & 12303 \\
$p 70$ & 49 & $9.25 E-07$ & 7256 \\
$p 71$ & 47 & $3.75 E-06$ & 9945 \\
$p 72$ & 46 & $9.68 E-06$ & 11952 \\
$p 73$ & 50 & 0.002756355 & 7505 \\
$p 74$ & 47 & 0.009871848 & 10485 \\
$p 75$ & 49 & 0.021730016 & 13178 \\
$p 76$ & 50 & 0.002644596 & 7776 \\
$p 77$ & 48 & 0.007916174 & 9692 \\
$p 79$ & 47 & 0.01986529 & 12681 \\
$p 80$ & 50 & 0.002472504 & 7409 \\
\hline
\end{tabular}

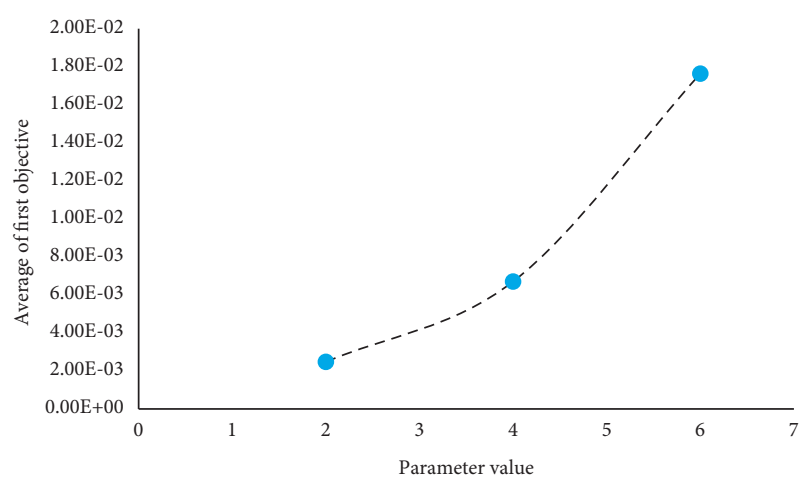

FIGURE 4: Impact of failure time parameter on the first objective function.

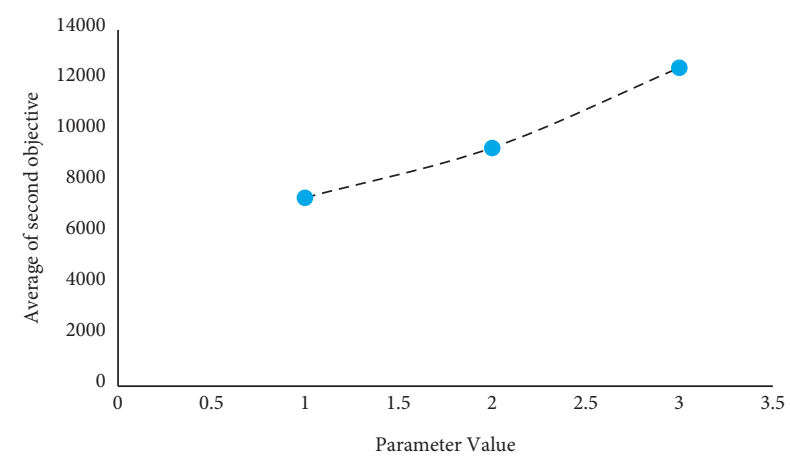

FIGURE 5: Impact of failure time on the second objective function.

relationship, the more allocated parts, the amount of reliability also increases. The same is true of the second objective function. As the failure time increases, the number of allocated parts increases, and therefore the total weight of the system increases. Therefore, according to Figure 5, the trend of the second objective function is ascending.

In Figures 6 and 7, the effect of the theta parameter or the Weibull distribution scale parameter on the first and second objective functions is investigated.

As shown in Figure 6, the larger the scale parameter, the lower the reliability of the entire system. The reason for this is that as the scale parameter increases, the reliability of each component decreases, and therefore the total reliability of the system decreases. But the effect of this parameter on the total weight of the system does not follow a specific trend. In Figure 7 , by increasing the scale parameter, first, the total weight of the system increases and then decreases. In other words, the second objective function is not directly affected by changes in this parameter.

In Figures 8 and 9, the effect of another parameter of the Weibull distribution, namely the beta or shape parameter, is investigated.

According to Figure 8, the higher the value of the figure parameter, the higher the reliability of the whole system. The reason for this is that as the shape parameter increases, the reliability of each component increases, and as a result, the reliability of the whole system increases. But this parameter does not have a significant effect on the heading function. This is also well seen in Figure 9. In general, it is concluded that Weibull distribution behavior does not have a direct effect on the relationship of the second objective function.

Finally, the effect of the maximum available budget parameter on the objective functions is investigated.

Figures 10 and 11 show that the higher the maximum budget, the more reliability moves towards greater values, and the maximum weight moves towards lower values. In other words, by increasing this parameter, both targets move towards better values. The reason for this is that with the increase in the maximum budget, the solution space of the 


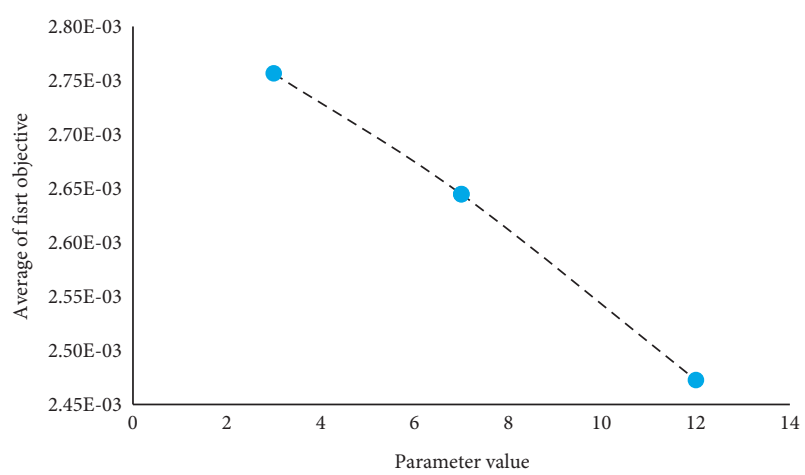

Figure 6: The effect of the scale parameter of the Weibull function on the first objective.

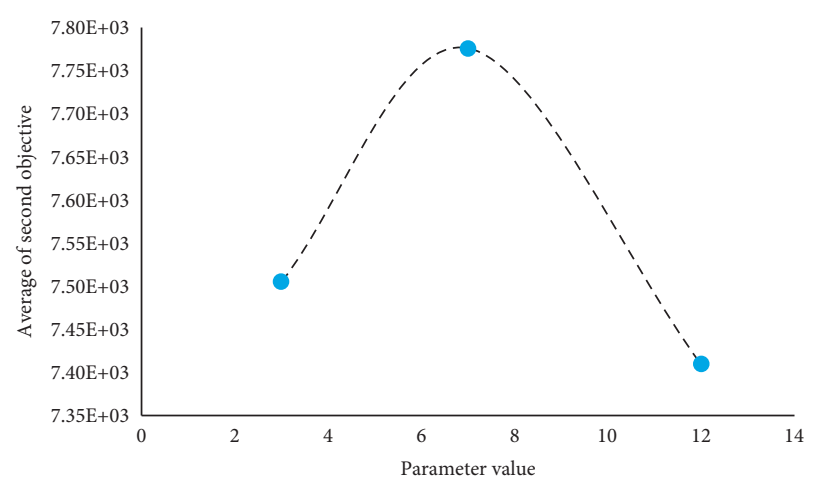

FIGURE 7: The effect of the scale parameter of the Weibull function on the second objective.

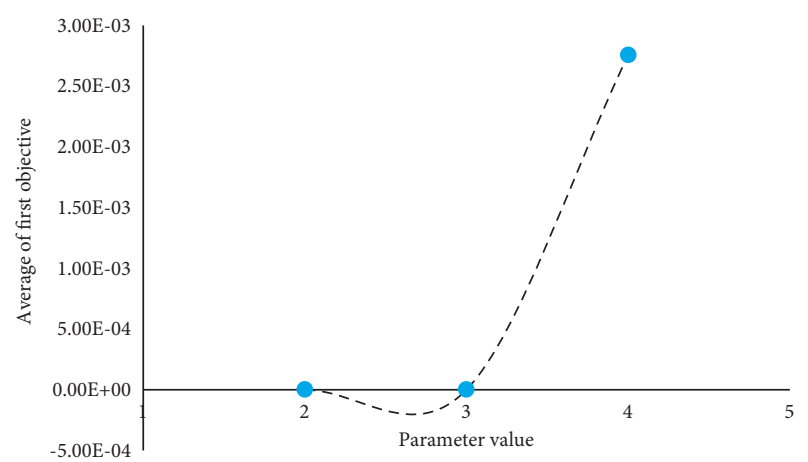

FIgURE 8: The effect of the shape parameter of the weibull function on the first objective.

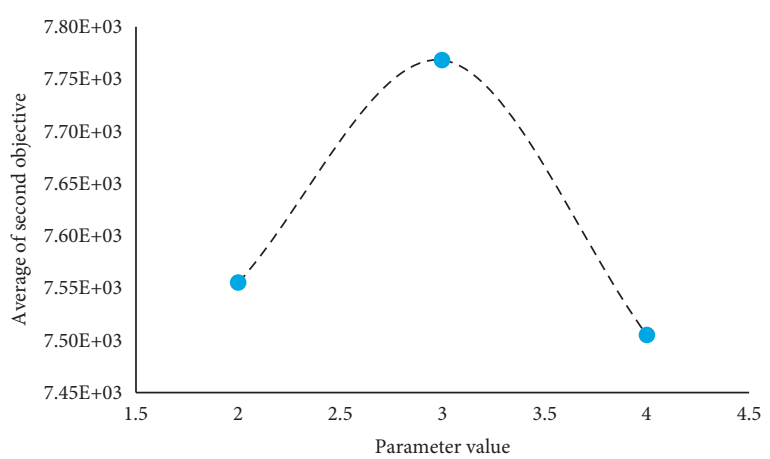

FIGURE 9: The effect of the shape parameter of the Weibull function on the second objective.

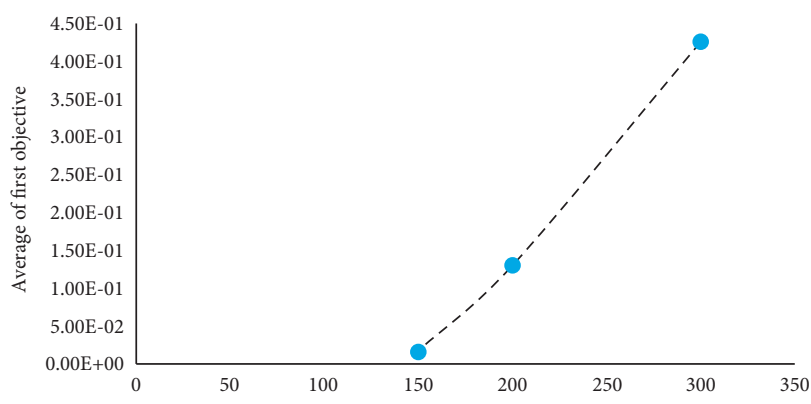

FIgURE 10: The effect of the maximum budget parameter on the first objective.

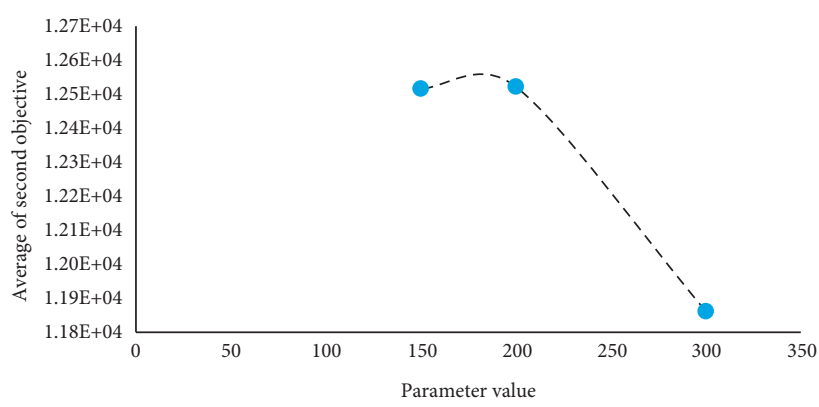

FIgURE 11: Impact of the maximum budget parameter on the second objective.

problem increases, and as a result, the objective functions can find better values for themselves.

\section{Conclusion}

In this research, first, the most critical research items in the field of redundancy allocation were reviewed. Next, it became clear that the issue of environmental pollution and sustainable maintenance repairs are two new and up-to-date topics for research in this field. Therefore, in this study, as a key innovation, we sought to provide a model that allocates redundancy in a complex system so that it covers environmental pollution and sustainable maintenance repairs well. In this regard, after a deep understanding of the basic articles, a developed model was designed for this purpose. Due to the high complexity of the model and its nonlinearity, designing a suitable and up-to-date approximate solution method for it was necessary. Therefore, the whale optimization, genetic and simulated annealing in a multiobjective mode was combined as a novel solution method.

In the numerical results, first, it was cleared that the objectives used in this research, i.e., maximizing the reliability of the system and reducing the weight of the whole system, are two completely conflicting ones. That is, increasing reliability leads to an increase in the weight of the entire system and vice versa. Therefore, optimizing these two objectives simultaneously leads to finding a set of optimal solutions, which we call the Pareto set of solutions. Next, 81 sample problems were designed and optimized by the proposed algorithm. The results of this section show that with increasing the failure time of each component, the 
reliability of the system increases, and also the weight of the whole system increases. Also, changing the Weibull distribution parameters has a direct effect on the total amount of system reliability but does not have a definite and accurate effect on the total weight of the system. Also, increasing the budget at hand for maintenance leads to finding solutions with more reliability and less weight.

The most important advantage of the proposed mathematical model of research is that it has been able to show the aspects of sustainability in the redundancy allocation problem and also has formulated repairs and maintenance of complex systems in a simple way. The designed metaheuristic algorithm also has the advantage of optimizing mathematical models with multiple objectives and variables in a short time. This issue can be considered as the most important managerial insight of this research. Another managerial insight of this research is that it is possible to manage the repair and maintenance planning of complex systems in such a way that in addition to reducing system costs, the productivity of manpower specialized in repair maintenance is increased.

In order to develop this research, the authors suggest considering uncertainty in the important parameters in the mathematical model and also using another novel metaheuristic algorithm such as the gray wolf optimizer and runner-root algorithm.

\section{Data Availability}

The input data for analysis of the mathematical method and metaheuristic algorithms which are used to support the findings of this study are available from the corresponding author upon request.

\section{Conflicts of Interest}

The authors declare that there are no conflicts of interest regarding the publication of this paper.

\section{References}

[1] A. Karevan and M. Vasili, "Sustainable reliability centered maintenance optimization considering risk attitude," Journal of Applied Research on Industrial Engineering, vol. 5, no. 3, pp. 205-222, 2018.

[2] X. Bei, N. Chatwattanasiri, D. W. Coit, and X. Zhu, "Combined redundancy allocation and maintenance planning using a two-stage stochastic programming model for multiple component systems," IEEE Transactions on Reliability, vol. 66, no. 3, pp. 950-962, 2017.

[3] Z. Ouyang, Y. Liu, S.-J. Ruan, and T. Jiang, "An improved particle swarm optimization algorithm for reliability-redundancy allocation problem with mixed redundancy strategy and heterogeneous components," Reliability Engineering \& System Safety, vol. 181, pp. 62-74, 2019.

[4] I. D. Lins and E. L. Droguett, "Redundancy allocation problems considering systems with imperfect repairs using multiobjective genetic algorithms and discrete event simulation," Simulation Modelling Practice and Theory, vol. 19, no. 1, pp. 362-381, 2011.
[5] Y.-C. Liang and M.-H. Lo, "Multiobjective redundancy allocation optimization using a variable neighborhood search algorithm," Journal of Heuristics, vol. 16, no. 3, pp. 511-535, 2010.

[6] F. Uysal, "An integrated model for sustainable performance measurement in supply chain," Procedia-Social and Behavioral Sciences, vol. 62, pp. 689-694, 2012.

[7] S. J. Sadjadi and R. Soltani, "An efficient heuristic versus a robust hybrid meta-heuristic for general framework of serial-parallel redundancy problem," Reliability Engineering \& System Safety, vol. 94, no. 11, pp. 1703-1710, 2009.

[8] A. Chambari, S. H. A. Rahmati, A. A. Najafi, and A. Karimi, "A bi-objective model to optimize reliability and cost of system with a choice of redundancy strategies," Computers \& Industrial Engineering, vol. 63, no. 1, pp. 109-119, 2012.

[9] M. K. Ghorabaee, M. Amiri, and P. Azimi, "Genetic algorithm for solving bi-objective redundancy allocation problem with k-out-of- $n$ subsystems," Applied Mathematical Modelling, vol. 39, no. 20, pp. 6396-6409, 2015.

[10] M. Abouei Ardakan, A. Zeinal Hamadani, and M. Alinaghian, "Optimizing bi-objective redundancy allocation problem with a mixed redundancy strategy," ISA Transactions, vol. 55, pp. 116-128, 2015.

[11] E. Zhang and Q. Chen, "Multiobjective reliability redundancy allocation in an interval environment using particle swarm optimization," Reliability Engineering \& System Safety, vol. 145, pp. 83-92, 2016.

[12] A. Salmasnia, H. Fallah Ghadi, and H. Mokhtari, "Simultaneous optimization of production and quality in a deterioration process," International Journal of Industrial Engineering \& Production Research, vol. 27, no. 3, pp. 273284, 2016.

[13] M. Teimoori, S. H. Edjtahed, and A. H. Niasar, "Design and simulation of Z-source inverter fed brushless DC motor drive supplied with fuel cell for automotive applications," Journal of Power Electronics \& Power Systems, vol. 6, no. 3, 2016.

[14] E. Long, S. Kokke, D. Lundie, N. Shaw, W. Ijomah, and C.-C. Kao, "Technical solutions to improve global sustainable management of waste electrical and electronic equipment (WEEE) in the EU and China," Journal of Remanufacturing, vol. 6 , no. 1, p. 1, 2016.

[15] A. Zaretalab, V. Hajipour, and M. Tavana, "Redundancy allocation problem with multi-state component systems and reliable supplier selection," Reliability Engineering \& System Safety, vol. 193, Article ID 106629, 2020.

[16] W. Wang, M. Lin, Y. Fu, X. Luo, and H. Chen, "Multiobjective optimization of reliability-redundancy allocation problem for multi-type production systems considering redundancy strategies," Reliability Engineering \& System Safety, vol. 193, Article ID 106681, 2020.

[17] L. Sahoo, A. K. Bhunia, and P. K. Kapur, "Genetic algorithm based multiobjective reliability optimization in interval environment," Computers \& Industrial Engineering, vol. 62, no. 1, pp. 152-160, 2012.

[18] S. Mirjalili and A. Lewis, "The whale optimization algorithm," Advances in Engineering Software, vol. 95, pp. 51-67, 2016. 